\title{
Multicritical points and topology-induced inverse transition in the random-field Blume-Capel model in a random network
}

\author{
R. Erichsen, Jr., ${ }^{*}$ Amanda Azevedo Lopes, ${ }^{\dagger}$ and S. G. Magalhaes ${ }^{\ddagger}$ \\ Instituto de Física, Universidade Federal do Rio Grande do Sul, Caixa Postal 15051, 91501-970 Porto Alegre, RS, Brazil
}

(Received 17 November 2016; revised manuscript received 24 March 2017; published 9 June 2017)

\begin{abstract}
The interplay between quenched disorder provided by a random field (RF) and network connectivity in the Blume-Capel (BC) model is the subject of this paper. The replica method is used to average over the network randomness. It offers an alternative analytic route to both numerical simulations and standard mean field approaches. The results reveal a rich thermodynamic scenario with multicritical points that are strongly dependent on network connectivity. In addition, we also demonstrate that the RF has a deep effect on the inverse melting transition. This highly nontrivial type of phase transition has been proposed to exist in the BC model as a function of network topology. Our results confirm that the topological mechanism can lead to an inverse melting transition. Nevertheless, our results also show that as the RF becomes stronger, the paramagnetic phase is affected in such way that the topological mechanism for inverse melting is disabled.
\end{abstract}

DOI: 10.1103/PhysRevE.95.062113

\section{INTRODUCTION}

The interplay between quenched disorder and many-spin interactions is a continual and challenging problem. For instance, it has been shown that the quenched disorder can replace a first order transition by a second one and suppress tricritical points [1-3]. Therefore, techniques such as the use of random networks [4] can be very helpful not only to check the predictions of others techniques, such as the standard mean field approach, but also because it can bring novel information about that difficult interplay.

The Blume-Capel (BC) model $[5,6]$ is one of the most widely studied spin models and the simplest one that presents a tricritical point. Renewed interest in this model is due to the recent discovery of numerous physical realizations within condensed-matter physics that exhibit inverse transitions (see Ref. [7] for a recent review). In this counterintuitive phase transition the ordered phase has higher entropy than the disordered one. The crystal field $D$ conjugated to the local quadratic three-state spin $\sigma(\sigma \in\{-1,0,1\})$ term in the Hamiltonian is a natural ingredient to produce a first order transition. It allows one to control the population of the active spin state, playing the role of a chemical potential and giving rise to a competition between the ferromagnetic $(\mathrm{F})$ phase $\left(m \equiv\left\langle\sigma_{i}\right\rangle \neq 0, Q \equiv\left\langle\sigma_{i}^{2}\right\rangle \neq 0\right)$ and the nonmagnetic (NM) phase ( $m=0, Q=0)$. In the standard mean field approach, at a certain value of $D$, the phase transition between $\mathrm{F}$ and $\mathrm{NM}$ presents a tricritical point $[5,8]$. This global phase diagram has been confirmed by other techniques (see, for instance, Ref. [9] and references therein).

The introduction of a random field (RF) following a bimodal distribution substantially increases the richness of the BC model. This can be seen in the standard mean field approach of the Random Field Blume-Capel (RFBC) model introduced by Kaufman and Kanner [10]. The phase diagrams of the RFBC model present tricritical points, a critical line, and,

\footnotetext{
*rubem@if.ufrgs.br

†amanda.azevedo4@gmail.com

${ }^{\ddagger}$ sgmagal@gmail.com
}

particularly, an isolated critical end point depending on the balance between the crystal field and the RF. Besides the NM phase, there is an onset of another type of paramagnetic $(\mathrm{P})$ phase $(m=0, Q \neq 0)$. Most importantly, there is also the onset of a novel phase at a sufficiently strong RF, the so-called ferromagnetic-nonmagnetic (F/NM) phase. The onset of this mixed $\mathrm{F} / \mathrm{NM}$ phase can be seen as a staggering manifestation of RF effects weakening the first order phase transition present in the $\mathrm{BC}$ model. However, could the existence of these multicritical points and the mixed phase F/NM also not be seen as an artifact of the standard mean field approach? In the random network approach, this standard mean field prediction, which corresponds to the limit of infinite connectivity, can be checked, since the network connectivity (the averaged coordination number) is a controllable parameter. It should be remarked that contrary to the Ghatak-Sherrington model [11], which has quenched bond disorder assuming both positive and negative values, the bonds in the $\mathrm{BC}$ model are always positive. The quenched disorder that we deal with in this work, beyond the RF, is that one associated with existence (or not) of a connection between two given sites. Since antiferromagnetic interactions are absent, this model does not present frustration. Therefore, we do not expect the existence of a spin-glass order. We also remark that our approach is essentially a mean field theory, in fact, a quite improved mean field theory as compared with the standard one since we can control the network connectivity. In that sense, our theory is also distinct from other recent approaches considering effects of bond randomness in the BC model with spatial dimensionality $d=2[12-14]$.

In addition, more recently, the standard $\mathrm{BC}$ model in a random network has also been studied, showing the existence of an inverse melting transition. This transition in which the $\mathrm{F}$ phase has higher entropy than the NM phase can be topologically induced, as suggested by De Martino and collaborators [15]. In other words, when the temperature is increased, a reentrant first order transition in the sequence NM-F phases can be observed depending on the network architecture. This topological mechanism becomes active only at sufficiently low spin connectivity. The large connectivity limit should come close to the standard mean field approach (infinite connectivity) with no reentrant transition in the BC 
model. In the case of the RFBC model, how does the RF affect the topological mechanism for inverse melting? Since the RF deeply affects the $\mathrm{P}$ phase in the RFBC model, one can expect that the topological mechanism for inverse transition may also be deeply affected.

The present work offers an alternative approach to study the RFBC model which can provide an answer to the question raised in the previous paragraphs. It is an extension to spin $S=1$ of finite connectivity techniques developed some years ago to study binary Ising spin glasses and related systems [4,16-20]. Since disorder is concerned with the random network, the replica method is applied. This method allows one to treat the quenched disorder given by the random network. As consequence, it is the free energy that has to be averaged rather than the partition function. The theoretical development conduces to a saddle-point equation for an order function, which can be solved self-consistently by a population dynamics technique. All physically relevant order parameters can be calculated through this order function.

The main question addressed in this paper concerns the interplay between quenched disorder (provided by the RF) and the network connectivity. The existence of multicritical points, the F/NM mixed phase, and the inverse melting transition will be tested depending on the network connectivity in the RFBC model. For instance, in our approach, the onset of the mixed phase $\mathrm{F} / \mathrm{NM}$ and multicritical points it is expected at large connectivity values as reported in the mean field approach for the RFBC model [10]. However, do they still appear at low connectivity values? The disappearance of the mixed phase $\mathrm{F} / \mathrm{NM}$ and some of the multicritical points at low connectivity values would indicate that their existence may be a mean field artifact. It should be noted that the answer to that question, particularly concerning the tricritical point, is also relevant to clarify the role of RF for the topology-induced inverse melting in the BC model.

The outline of the paper is as follows. In the next section the finite-connectivity replica theory for the BC model with a random field is developed. In Sec. 3 results for the relevant order parameters, free energy, and phase diagrams for some representative values of the mean connectivity are presented discussed. The paper ends with a summary and concluding remarks.

\section{MODEL AND REPLICA PROCEDURE}

A system of $N$ interacting three-state Ising spins $\sigma_{i}, i=$ $1, \ldots, N$, as described by the Hamiltonian, was considered:

$$
H=-\frac{J}{c} \sum_{i<j} \sigma_{i} c_{i j} \sigma_{j}+D \sum_{i} \sigma_{i}^{2}+\sum_{i} \theta_{i} \sigma_{i}
$$

The $c_{i j}$ are independent, identically distributed random variables (i.i.d.r.v) indicating if a given pair of spins $(i, j)$ are connected $\left(c_{i j}=1\right)$ or not $\left(c_{i j}=0\right)$. We assume that the connections are bidirectional, i.e., $c_{i j}=c_{j i}$. They are chosen according to the distribution

$$
p\left(c_{i j}\right)=\frac{c}{N} \delta_{c_{i j}, 1}+\left(1-\frac{c}{N}\right) \delta_{c_{i j}, 0},
$$

where the connectivity $c$, the average number of connections per spin, remains finite in the thermodynamic limit, such that $\lim _{N \rightarrow \infty} c / N=0$. The connected sites interact through a $\mathrm{F}$ coupling $J>0$. The crystal field favors the population of the zero state, if $D>0$, or the active states $\sigma= \pm 1$, if $D<0$. If $D$ is sufficiently large and negative, the binary Ising model with finite connectivity and spins $\sigma_{i} \in\{-1,1\}$ is retrieved [21]. The local fields $\theta_{i}$ are i.i.d.r.v., chosen according to a bimodal distribution

$$
p\left(\theta_{i}\right)=\frac{1}{2} \delta\left(\theta_{i}-\theta\right)+\frac{1}{2} \delta\left(\theta_{i}+\theta\right),
$$

where $\theta$ is the local field amplitude.

Although the coupling $J$ is assumed to be constant, the random nature of the connectivity variable $c_{i j}$ introduces a quenched disorder. In order to obtain the thermodynamic properties of the system, the disorder-averaged free energy is calculated through the standard replica procedure,

$$
f(\beta)=-\lim _{N \rightarrow \infty} \frac{1}{\beta N} \lim _{n \rightarrow 0} \frac{1}{n} \log \left\langle Z^{n}\right\rangle,
$$

where

$$
Z=\sum_{\sigma_{1} \ldots \sigma_{N}} \mathrm{e}^{-\beta H}
$$

is the partition function. The angle brackets in Eq. (4) stand for the disorder average. In the limit $c / N \rightarrow 0$, the disorderaverage replicated partition function becomes

$$
\begin{aligned}
\left\langle Z^{n}\right\rangle & =\sum_{\boldsymbol{\sigma}^{1} \ldots \boldsymbol{\sigma}^{n}}\left\langle\operatorname { e x p } \left[\beta \sum_{i, \alpha} \theta_{i} \sigma_{i}^{\alpha}-\beta D \sum_{i, \alpha}\left(\sigma_{i}^{\alpha}\right)^{2}\right.\right. \\
& \left.\left.+\frac{c}{2 N} \sum_{i \neq j}\left(\mathrm{e}^{\frac{\beta J}{c} \sum_{\alpha} \sigma_{i}^{\alpha} \sigma_{j}^{\alpha}}-1\right)\right]\right\rangle_{\left\{\theta_{i}\right\}},
\end{aligned}
$$

with $\alpha=1, \ldots, n$ denoting the replica index, and $\sigma^{\alpha}$ being the a vector with $N$ components representing the state of replica $\alpha$. Note that, since the connectivity $c$ is finite, one cannot expand the inner exponential and introduce the order parameters, like in standard infinite-connectivity calculations. Instead, in order to extract the variables into summation from the inner exponential, we follow Ref. [4] and introduce the order function $P(\sigma)$, which represents the fraction of sites with the replica configuration $\sigma$ and obtain the free energy (see the Appendix)

$$
\begin{aligned}
f(\beta)= & -\lim _{n \rightarrow 0} \frac{1}{\beta n}\left[-\frac{c}{2} \sum_{\boldsymbol{\sigma} \boldsymbol{\tau}} P(\boldsymbol{\sigma}) P(\boldsymbol{\tau})\left(\mathrm{e}^{\frac{1}{c} \beta J \sum_{\alpha} \sigma_{\alpha} \tau_{\alpha}}-1\right)\right. \\
& +\log \sum_{\boldsymbol{\sigma}}\left\langle\operatorname { e x p } \left[\beta \theta \sum_{\alpha} \sigma_{\alpha}-\beta D \sum_{\alpha} \sigma_{\alpha}^{2}\right.\right. \\
& \left.\left.\left.+c \sum_{\boldsymbol{\tau}} P(\boldsymbol{\tau})\left(\mathrm{e}^{\frac{1}{c} \beta J \sum_{\alpha} \sigma_{\alpha} \tau_{\alpha}}-1\right)\right]\right\rangle_{\theta}\right],
\end{aligned}
$$

with $P(\sigma)$ given by the self-consistent relationship

$$
\begin{aligned}
P(\boldsymbol{\sigma})= & \mathcal{N}^{-1}\left\langle\operatorname { e x p } \left[\beta \theta \sum_{\alpha} \sigma_{\alpha}-\beta D \sum_{\alpha} \sigma_{\alpha}^{2}\right.\right. \\
& \left.\left.+c \sum_{\boldsymbol{\tau}} P(\boldsymbol{\tau})\left(\mathrm{e}^{\frac{\beta J}{c} \sum_{\alpha} \sigma_{\alpha} \tau_{\alpha}}-1\right)\right]\right\rangle_{\theta},
\end{aligned}
$$


where

$$
\begin{aligned}
\mathcal{N}= & \sum_{\sigma}\left\langle\operatorname { e x p } \left[\beta \theta \sum_{\alpha} \sigma_{\alpha}-\beta D \sum_{\alpha} \sigma_{\alpha}^{2}\right.\right. \\
& \left.\left.+c \sum_{\boldsymbol{\tau}} P(\boldsymbol{\tau})\left(\mathrm{e}^{\frac{\beta J}{c} \sum_{\alpha} \sigma_{\alpha} \tau_{\alpha}}-1\right)\right]\right\rangle_{\theta}
\end{aligned}
$$

is a normalization factor.

Our search for solutions of Eq. (8) will be restricted to the replica symmetry (RS) ansatz, [4,17]. This means that $P(\sigma)$ should remain invariant under replica permutations, and, consequently, for three-state spins, it should depend only on the summations $\sum_{\alpha} \sigma_{\alpha}$ and $\sum_{\alpha} \sigma_{\alpha}^{2}$ Thus, in extension of the RS ansatz for the order function in finite-connectivity twostate Ising models [21], for the three-state model we follow Ref. [22] and assume

$$
P(\boldsymbol{\sigma})=\int \mathcal{D} W \frac{\mathrm{e}^{\beta h \sum_{\alpha} \sigma_{\alpha}-\beta \tilde{h} \sum_{\alpha} \sigma_{\alpha}^{2}}}{\chi_{0}^{n}(h, \tilde{h})}
$$

for any real $n$, where $\mathcal{D} W \equiv d h d \tilde{h} W(h, \tilde{h}), h$ and $\tilde{h}$ are the two components of the local field, $W(h, \tilde{h})$ is a density which has to be determined self-consistently, and

$$
\chi_{\sigma}(h, \tilde{h})=1+2 \mathrm{e}^{-\beta \tilde{h}} \cosh \left[\beta\left(h+\frac{J}{c} \sigma\right)\right] .
$$

Introducing this ansatz in Eq. (8), expanding the exponential, summing over $\tau_{\alpha}^{l}$, and using the identity $\sum_{\sigma} \delta_{\sigma \sigma_{\alpha}}=1$ in order to extract the appropriate dependence on $\sum_{\alpha} \sigma_{\alpha}$ and $\sum_{\alpha}\left(\sigma_{\alpha}\right)^{2}$, we obtain

$$
\begin{aligned}
P(\boldsymbol{\sigma})= & \left\langle\mathrm{e}^{\beta \theta \sum_{\alpha} \sigma_{\alpha}-\beta D \sum_{\alpha} \sigma_{\alpha}^{2}}\right\rangle_{\theta} \sum_{k=0}^{\infty} \frac{\mathrm{e}^{-c} c^{k}}{k !} \\
& \times \int \prod_{l=1}^{k} \mathcal{D} W_{l} \frac{\mathrm{e}^{\sum_{\alpha} \sum_{\sigma} \delta_{\sigma \sigma_{\alpha}} \log \chi_{\sigma}\left(h_{l}, \tilde{h}_{l}\right)}}{\chi_{0}^{n}\left(h_{l}, \tilde{h}_{l}\right)} .
\end{aligned}
$$

For three-state spins we use the representation

$$
\delta_{\sigma \sigma_{\alpha}}=1-\sigma^{2}-\sigma_{\alpha}^{2}+\sigma \sigma_{\alpha} / 2+3 \sigma^{2} \sigma_{\alpha}^{2} / 2
$$

Summing over $\sigma$ and introducing the RS ansatz on the lefthand side of Eq. (12) we obtain a self-consistent relationship for $W(h, \tilde{h})$,

$$
\begin{aligned}
W(h, \tilde{h})= & \sum_{k=0}^{\infty} \frac{\mathrm{e}^{-c} c^{k}}{k !}\left\langle\int \prod_{l=1}^{k} \mathcal{D} W_{l} \delta\left[h-\theta-\frac{1}{\beta} \sum_{l=1}^{k} \phi\left(h_{l}, \tilde{h}_{l}\right)\right]\right. \\
& \left.\times \delta\left[\tilde{h}-D+\frac{1}{\beta} \sum_{l=1}^{k} \psi\left(h_{l}, \tilde{h}_{l}\right)\right]\right\rangle_{\theta}
\end{aligned}
$$

where

$$
\phi(h, \tilde{h})=\frac{1}{2} \log \frac{\chi_{+1}(h, \tilde{h})}{\chi_{-1}(h, \tilde{h})}
$$

and

$$
\psi(h, \tilde{h})=\frac{1}{2} \log \frac{\chi_{+1}(h, \tilde{h}) \chi_{-1}(h, \tilde{h})}{\chi_{0}^{2}(h, \tilde{h})} .
$$

To determine the density $W(h, \tilde{h})$ we proceed numerically by means of population dynamics of a large number of fields updated as follows. First, a number $k$ is chosen from a Poisson distribution of mean $c$. Then cells $\left(h_{l}, \tilde{h}_{l}\right)$ with $l$ running from 1 to $k$ are selected at random from the population, and the summations in the delta functions are calculated. Next, one selects at random a new cell $(h, \tilde{h})$ from the population and sets

$$
\begin{aligned}
& h=\theta+\frac{1}{\beta} \sum_{l=1}^{k} \phi\left(h_{l}, \tilde{h}_{l}\right), \\
& \tilde{h}=D-\frac{1}{\beta} \sum_{l=1}^{k} \psi\left(h_{l}, b_{l}\right)
\end{aligned}
$$

continuing the procedure until it converges to a limiting $W(h, \tilde{h})$.

The relevant order parameters for this problem are the per site magnetization $m \equiv\langle\sigma\rangle$ and the correlation parameter $Q \equiv$ $\left\langle\sigma^{2}\right\rangle$. In the replica symmetric approach they are calculated through the knowledge of $W(h, \tilde{h})$ :

$$
m=\int \mathcal{D} W\langle\sigma\rangle
$$

and

$$
Q=\int \mathcal{D} W\left\langle\sigma^{2}\right\rangle
$$

where

$$
\langle\sigma\rangle=\frac{2 \mathrm{e}^{-\beta \tilde{h}} \sinh (\beta h)}{1+2 \mathrm{e}^{-\beta \tilde{h}} \cosh (\beta h)}
$$

and

$$
\left\langle\sigma^{2}\right\rangle=\frac{2 \mathrm{e}^{-\beta \tilde{h}} \cosh (\beta h)}{1+2 \mathrm{e}^{-\beta \tilde{h}} \cosh (\beta h)} .
$$

As usual, $m \neq 0$ indicates magnetic ordering, $m=0, Q \neq 0$ are $\mathrm{P}$, and $m=0=Q$ are NM.

The free-energy has energetic and entropic contributions, $f=f_{\text {en }}+f_{\text {entr }}$, that are, respectively, the first and second terms of the right-hand side of Eq. (7). Introducing the RS ansatz and considering that the replicas are noninteracting, the energy contribution can be written, in the $n \rightarrow 0$ limit, as

$$
f_{\mathrm{en}}(\beta)=\frac{c}{2 \beta} \int \mathcal{D} W \mathcal{D} W^{\prime} \log \mathcal{U}\left(h, \tilde{h}, h^{\prime}, \tilde{h}^{\prime}\right),
$$

where

$$
\begin{aligned}
\mathcal{U}\left(h, \tilde{h}, h^{\prime}, \tilde{h^{\prime}}\right) \\
=\left(1+2 \mathrm{e}^{-\beta\left(\tilde{h}+\tilde{h^{\prime}}\right)}\left\{\mathrm{e}^{\frac{\beta J}{c}} \cosh \left[\beta\left(h+h^{\prime}\right)\right]\right.\right. \\
\left.+\mathrm{e}^{-\frac{\beta J}{c}} \cosh \left[\beta\left(h-h^{\prime}\right)\right]\right\}+2 \mathrm{e}^{-\beta \tilde{h}} \cosh (\beta h) \\
\left.\quad+2 \mathrm{e}^{-\beta \tilde{h^{\prime}}} \cosh \left(\beta h^{\prime}\right)\right) \chi_{0}^{-1}(h, \tilde{h}) \chi_{0}^{-1}\left(h^{\prime}, \tilde{h}^{\prime}\right) .
\end{aligned}
$$

The entropy contribution becomes, in the $n \rightarrow 0$ limit,

$$
f_{\text {entr }}=-\frac{1}{\beta} \sum_{k} \frac{\mathrm{e}^{-c} c^{k}}{k !} \int \prod_{l} \mathcal{D} W_{l}\left\langle\log \mathcal{S}\left(\left\{h_{l}\right\},\left\{\tilde{h}_{l}\right\}, \theta\right)\right\rangle_{\theta},
$$


where

$$
\begin{aligned}
\mathcal{S}\left(\left\{h_{l}\right\},\left\{\tilde{h}_{l}\right\}, \theta\right)= & 1+\mathrm{e}^{-\beta(D-\theta)} \prod_{l} \frac{\chi_{+1}\left(h_{l}, \tilde{h}_{l}\right)}{\chi_{0}\left(h_{l}, \tilde{h}_{l}\right)} \\
& +\mathrm{e}^{-\beta(D+\theta)} \prod_{l} \frac{\chi_{-1}\left(h_{l}, \tilde{h}_{l}\right)}{\chi_{0}\left(h_{l}, \tilde{h}_{l}\right)} .
\end{aligned}
$$

\section{RESULTS AND DISCUSSION}

The implementation of population dynamics requires an initial guess for $W(h, \tilde{h})$. A population of $N$ two-component fields was distributed in two distinct ways: (1) the components $h$ and $\tilde{h}$ of each field being randomly chosen satisfying $0 \leqslant h<1$ and $-1<\tilde{h} \leqslant 0$, corresponding to an $\mathrm{F}$ initial condition; and (2) for each field $h=0$ and $-1<\tilde{h}<0$, corresponding to a $\mathrm{P}$ initial condition. For each set of parameters $c, D / J, \theta / J$ and $T / J$ the population dynamics is allowed to run until a stationary distribution $W(h, \tilde{h})$ is reached.

Illustrative examples of stationary distributions for $c=5$, $D / J=0.6$, and $T / J=0$ are shown in Fig. 1 . The population size was $N=1 \times 10^{5}$ fields. Figure 1(a) shows the stationary distribution for $\theta / J=0.2$. This narrow and symmetric distribution corresponds to a NM phase, with $m=0$ and $Q \approx 0$. Figure 1 (b) shows the stationary distribution for $\theta / J=$ 0.4 . This wider and asymmetric distribution corresponds to the $\mathrm{F}$ phase, with $m \neq 0$ and $Q \neq 0$. Figure 1(c) shows the stationary distribution for $\theta / J=0.6$. This wide and symmetric distribution corresponds to the P phase with $m=0$ and $Q \neq 0$.

Before discussing the phase diagrams, it is worth showing some examples of curves for the order parameters and free energy as functions of the temperature. Figure 2(a) shows $m, Q$, and $f$ versus $T / J$ at constant $\theta / J=0.25$ and $c=5$. There are two regimes, depending on $D / J$. At high $D / J$, e.g., $D / J=0.6$, and low $T / J$, the magnetization is zero and the correlation parameter is small, as shown in Fig. 2(a). This is the NM phase, reported in Ref. [10] for the fully connected network. The reason for the presence of this phase is that the high value of $D / J$ favors the local NM state $\sigma=0$. As the temperature increases, there is a discontinuous transition to an F phase. At constant $\theta / J=0.25$ the thermodynamic transition is located at $T / J \approx 0.093$, a value that is found by the crossing of the NM and F free energies, as shown in the bottom panel of Fig. 2(a). This means that the thermal noise is capable of disrupting the network symmetry and giving rise to an F phase, where both $m$ and $-m$ magnetizations are equally probable. We will return to this subject later in this paper. When the thermal noise becomes too large the $\mathrm{F}$ phase is destroyed, giving rise to a $\mathrm{P}$ phase, with zero $m$ and large $Q$. The continuous transition, for $\theta / J=0.25$, is located at $T / J \approx 0.256$. Incidentally, at low $D / J$, as the temperature increases, there is only a continuous F-P transition, e.g., $T / J \approx 0.546$ at $D / J=0.25$ (not shown in the figure). The critical exponent for the continuous F-P transition does not depend on the value of $c$, being consistent with a mean field theory, i.e., $\beta=0.5$. This is because our approach allows us to control the average connectivity, but the length scale of the interactions remains the same as the standard mean field theory.

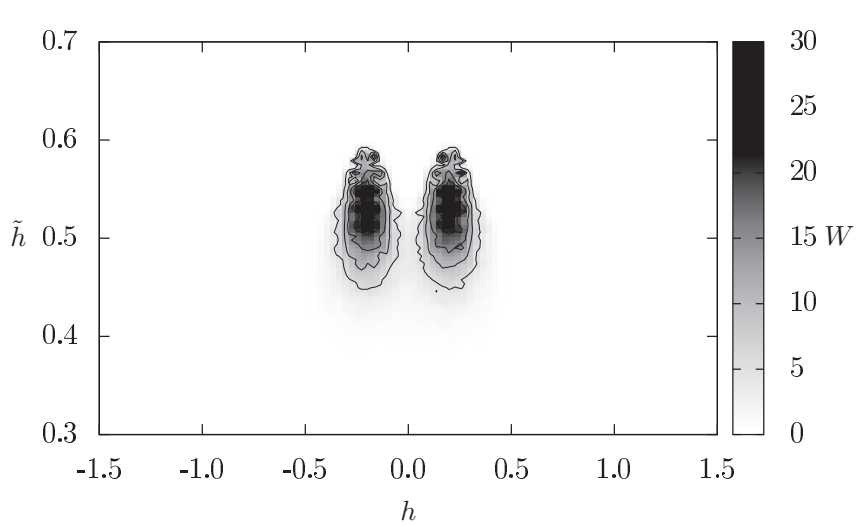

(a)

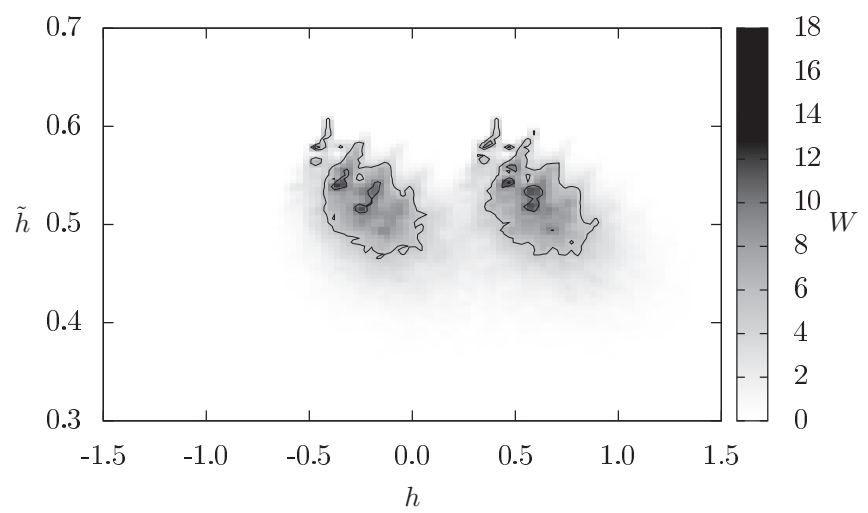

(b)

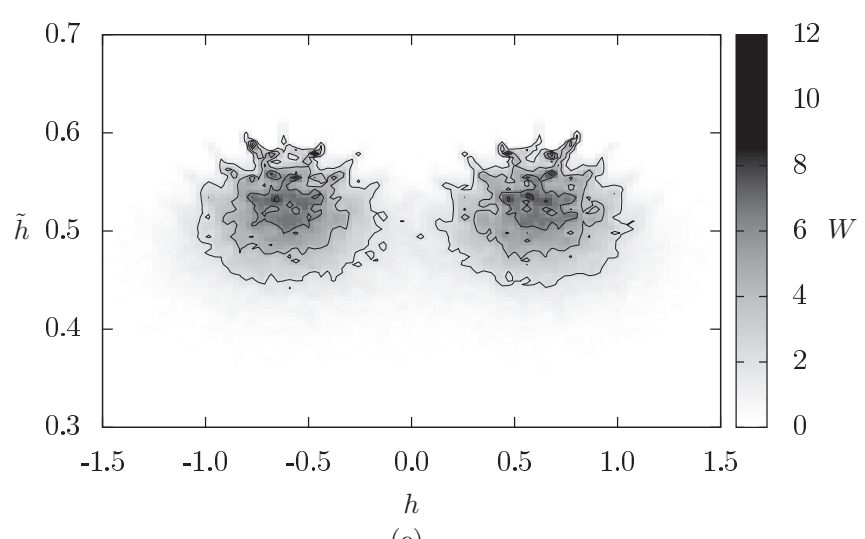

(c)

FIG. 1. Local field distribution $W(h, \tilde{h})$ for $c=5, D=0.6$, and $T=0.2$, for (a) $\theta=0.2$ (NM phase), (b) $\theta=0.4$ (F phase), and (c) $\theta=0.6$ ( $\mathrm{P}$ phase $)$.

Figure 2(b) shows curves of the order parameters and free energy versus RF amplitude $\theta / J$ at constant $T / J=0.02$, $D / J=0.4$ for two representative values of $c$. For $c=10$, as $\theta / J$ increases, there is, first, a smooth decrease in the magnetization $m$ to a lower plateau and then a continuous F-P transition at $\theta / J \approx 0.6$. Conversely, for a larger $c=25$ there are two discontinuous transitions as $\theta / J$ increases. First, there is a transition from a large $m \mathrm{~F}$ phase to a lower $m, \mathrm{~F} / \mathrm{NM}$ phase at $\theta / J \approx 0.362$. This lower $m \mathrm{~F}$ phase is presented in Ref. [10] as the F/NM phase, and it is argued there that it would remain a distinct phase even for low-connectivity three-dimensional systems. Here we show that this phase is 


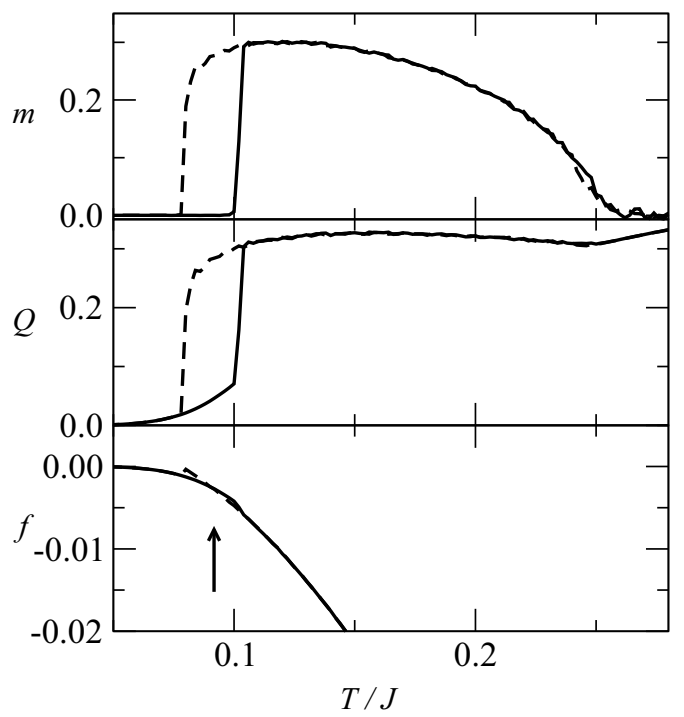

(a)

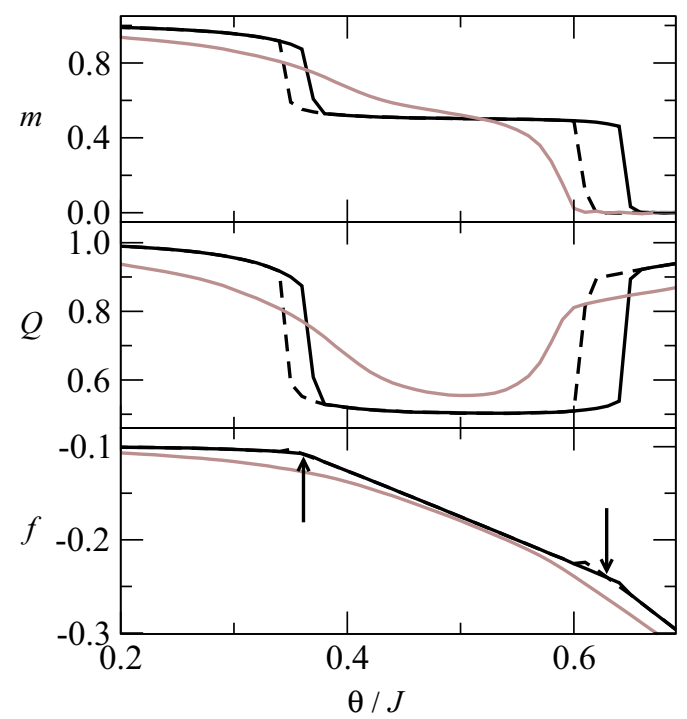

(b)

FIG. 2. (a) Per-site magnetization (top), correlation parameter (middle), and per-site free energy (bottom) versus temperature, with $c=5, \theta / J=0.25$, and $D / J=0.6$ for increasing and decreasing $T$ in solid and dashed lines, respectively. The arrow indicates the discontinuous thermodynamic transition. (b) Per-site magnetization (top), correlation parameter (middle), and per-site free energy (bottom) versus random-field amplitude, with $c=25$ (black lines) or $c=10$ (brown lines), $T / J=0.02$, and $D / J=0.4$ for increasing and decreasing $\theta$ in solid and dashed lines, respectively. The arrow indicates the discontinuous thermodynamic transition.

a mean field artifact which is suppressed already for $c=10$. As $\theta / J$ increases further, for $c=25$, there is a discontinuous $\mathrm{F} / \mathrm{NM}-\mathrm{P}$ transition at $\theta / J \approx 0.629$.

\section{A. Zero-temperature phase diagrams}

Phase diagrams at zero temperature are elucidative of the role that is played by network connectivity. Figure 3 shows the $D / J$ versus $\theta / J$ phase diagram for $c=5, c=10$, and

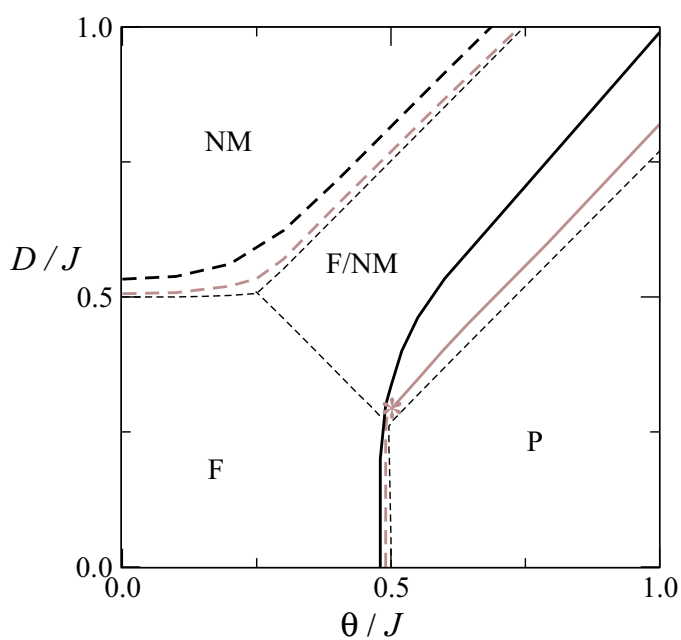

FIG. 3. Phase diagram $D / J$ vs $\theta / J$ at $T=0$ for $c=5$ (black heavy lines), $c=10$ (brown heavy lines), and $c=25$ (black light lines). Continuous and discontinuous phase boundaries are represented, respectively by solid and dashed lines. NM, F, P, and F/NM represent, respectively, nonmagnetic, ferromagnetic, paramagnetic and ferromagnetic-nonmagnetic phases. The asterisk indicates a tricritical point, over the F-P transition, for $c=10$.

$c=25$. There are four thermodynamic phases. First, at a small $D / J$ and $\theta / J$ the system organizes in ferromagnetic phase $\mathrm{F}$. In this region, the landscape is dominated by ferromagnetic couplings. The crystal field $D$ favors the NM state $\sigma=0$ and destroys the $\mathrm{F}$ ordering. Consequently, as $D / J$ increases, a NM phase appears. The transition between F and NM is discontinuous. Conversely, the bimodal $\mathrm{RF} \pm \theta$ favors the active states $\sigma= \pm 1$. The ferromagnetic ordering also is destroyed, and a $\mathrm{P}$ phase appears as the RF amplitude $\theta / J$ increases. The F-P transition is continuous for $c=5$, but for $c=10$ it shows a tricritical point located at $D_{\text {tric }} / J \approx 0.3$ and $\theta_{\text {tric }} / J \approx 0.507$, indicated by an asterisk in the figure. The transition is discontinuous at a small $D / J$ and continuous otherwise. It is not shown in the figure, but the tricritical point displaces upwards through the F-P transition line as $c$ increases. At $c=25$, this critical point is beyond the investigated range, approaching the full connectivity limit presented in Ref. [10], where this transition is fully discontinuous. The figure also shows that the $\mathrm{F} / \mathrm{NM}$ phase is a diagonal region between $\mathrm{NM}$ and $\mathrm{P}$ phases when $D / J$ and $\theta / J$ are both large. It is important to stress that, according to Fig. 2(b), this is not a distinct phase from the $\mathrm{F}$ phase for $c=5$ and $c=10$. Only for $c=25$ is there a discontinuous transition, indicated by the light dashed line. The crystal field can be negative, and the F-P transition lines extend vertically to the negative $D / J$ region.

It is worth to discuss the underlying physical mechanisms that are responsible for the strong dependence on the average connectivity $c$ presented by the the nature of the F-P transition. It is possible to explain this dependence by looking to the local field on site $i$ :

$$
h_{i}=\frac{J}{2 c} \sum_{j} c_{i j} \sigma_{j}-D \sigma_{i}+\theta_{i}
$$


The spin at site $i$ adjusts its state according to $h_{i}$. For small $D$, there is a competition between the first and the third terms of $h_{i}$. The first term on $h_{i}$ is prone to quenched disorder through the random variable $c_{i j}$. For large $c$, the fluctuations in the first term induced by the random connectivity are very small, and the F phase remains as the ground state provided $\theta / J<0.5$ [10]. At $\theta / J=0.5$ the system undergoes an abrupt transition to the $\mathrm{P}$ phase because the third term, i.e., the RF, becomes larger than the $\mathrm{F}$ interaction at every site. This picture changes when $c$ is small. In this case, the fluctuations in the first term increase due to the quenched disorder, favoring a gradual transition to the $\mathrm{P}$ phase. This is an evidence that the disorder softens the transitions. Furthermore, the crystal field $D$ favors the NM state $\sigma=0$. For small $\theta / J$, the second term in Eq. (27) becomes dominant for $D / J \gtrsim 0.5$ and, therefore, produces the NM phase.

The disappearance of the mixed F/NM phase boundary in the low-connectivity regime can be also undertood from the Eq. (27) when both $D / J$ and $\theta / J$ are sufficiently large. In this situation, because the RF distribution is symmetric [see Eq. (3)], the system organizes in a way that half of the sites align parallel to one of the two possible direction of the RF and the other half assumes the state $\sigma=0$. This results, at small temperature, $m \approx \pm 1 / 2$ and $Q \approx \pm 1 / 2$, as shown in Fig. 2(b). For a sufficiently large connectivity, like $c=25$, $\mathrm{F} / \mathrm{NM}$ and $\mathrm{F}$ are distinct phases, while for low to moderate, e.g., $c=10$, there is only a smooth decrease on the magnetization. The smoothing of the transition also results from the disorder, which becomes more important the smaller the connectivity.

\section{B. Finite-temperature phase diagrams}

Temperature effects will be discussed in terms of $T / J$ versus $\theta / J$ phase diagrams for several values of $D / J$ and connectivity $c$.

The $T / J$ versus $\theta / J$ phase diagram for the lowconnectivity regime $c=5$ is presented in Fig. 4(a) for three representative values of the crystal field, $D / J=0.25$, 0.56 , and 0.60 . For $D / J=0.25$, onset of the $\mathrm{F}$ phase occurs in the region below $T / J \approx 0.60$ and $0 \leqslant \theta / J \lesssim 0.49$. The $\mathrm{P}$ phase appears in the remaining phase diagram. The transition between these two phases, the F-P transition, is continuous. For increasing $D / J$ there is a rapidly evolving change in the shape of the phase diagram. In the range $0.5 \lesssim D / J \lesssim 0.6$, the crystal field $D$ favors the local state $\sigma=0$. As a consequence, for $D / J=0.56$, the NM phase appears at a low $T / J$ and small $\theta / J$. Then there is a NM-F discontinuous transition. For a higher $T / J$ and larger $\theta / J$, the F-P transition remains continuous. However, for $D / J=0.6$, the F phase is completely suppressed at a small $\theta / J$.

Nevertheless, for intermediate values of $\theta / J$, the continuous transition NM-F becomes discontinuous at lower temperatures with a tricritical point located at $\theta_{\text {tric }} / J=0.231$ and $T_{\text {tric }} / J=0.150$. Most importantly, there is a strong reentrance in the discontinuous part of the NM-F transition indicating the onset of an inverse melting transition. In contrast, at a large $\theta / J$, the F-P transition is not affected. It remains continuous and nonreentrant. The phase diagrams for $c=10$ are shown

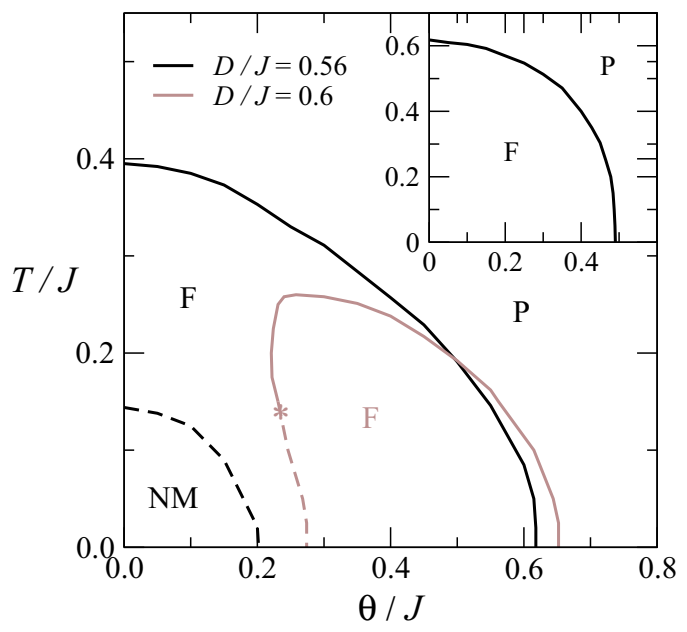

(a)

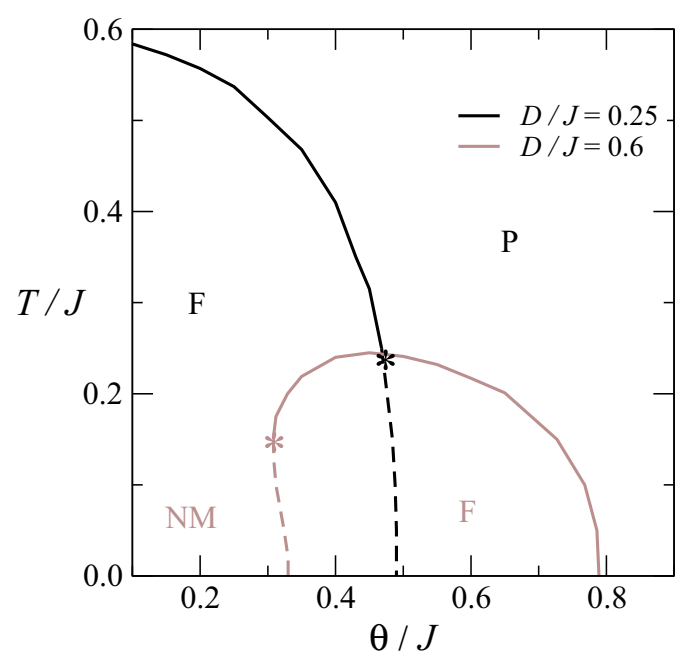

(b)

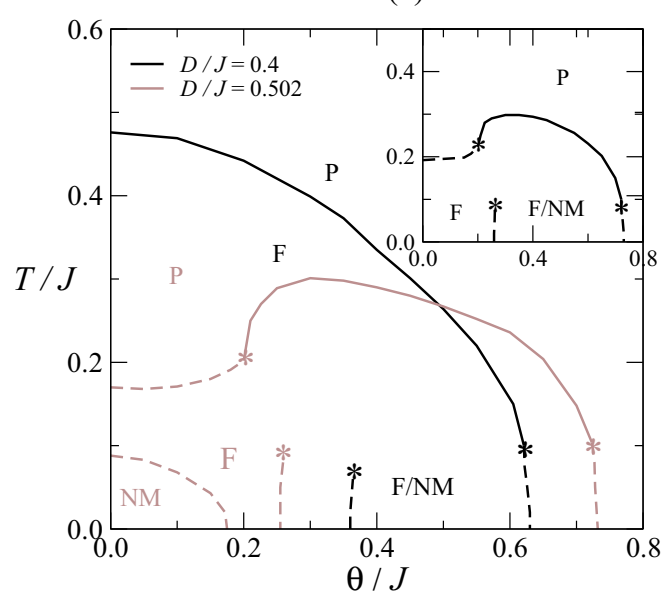

(c)

FIG. 4. (a) Phase diagram $T / J$ vs $\theta / J$ for $c=5$, for $D / J=$ $0.56, D / J=0.6$, and $D / J=0.25$ (in the inset). (b) Phase diagram $T / J$ vs $\theta / J$ for $c=10$, for $D / J=0.25$ and $D / J=0.6$. (c) Phase diagram $T / J$ vs $\theta / J$ for $c=25$, for $D / J=0.4$ and $D / J=0.502$. In the inset, $D / J=0.5$. Solid and dashed lines represent continuous and discontinuous phase boundaries, respectively.

in Fig. 4(b). For $D / J=0.25$, the F-P transition becomes discontinuous at lower temperatures with the emergence of 
a tricritical point located at $\theta_{\text {tric }} / J=0.468$ and $T_{\text {tric }} / J=$ 0.252 . Furthermore, for $D / J=0.60$, reentrance in the NM-F transition is less pronounced.

More expressive changes are observed in Fig. 4(c) for a larger connectivity, e.g., $c=25$ for $D / J=0.4,0.5$ (see the inset), and 0.502 . This is a large connectivity value that allows the appearance of most of the features observed in the fully connected network regime $(c \rightarrow \infty)$. For $D / J=0.4$, the phase diagram is basically the same as already found in the Fig. 4(b). The main difference is concerned with the location of the tricritical point. The phase diagrams start to become very sensitive to crystal field changes at $D / J \gtrsim 0.50$. For $D / J=0.50$, the F-P transition becomes discontinuous at a low $\theta / J$ with a tricritical point located at $T / J \approx 0.22$ and $\theta / J \approx 0.22$. Moreover, as $\theta / J$ enhances, a critical line appears between $\mathrm{F}$ and the mixed $\mathrm{F} / \mathrm{NM}$ phases, then an $\mathrm{F} / \mathrm{NM}-\mathrm{P}$ discontinuous transition and a second tricritical point at $T / J \approx 0.08$ and $\theta / J \approx 0.72$. For a slightly higher value, $D / J=0.502$, the most important qualitative difference compared to the previous case is that the NM phase appears at a low temperature. This phase increases quickly, so that already at $D / J=0.503$ the $\mathrm{F}$ phase is suppressed at a low $\theta / J$. It should be noted that the increase of the NM phase prevents the appearance of the critical end point observed in the mean field approximation (see Ref. [10]).

It is possible to explain the reentrant behavior found in the Figs. 4(a) and 4(b) by a competition between the crystal field and the thermal noise, for a given RF amplitude $\theta$. In the reentrant region, at a low temperature, the crystal field dominates, favoring inactive state $\sigma=0$. Here we extend the argument recently proposed in Ref. [15] to include RF effects. In the random network topology with moderate to low mean connectivity, a fraction of the nodes have a very low degree. We consider a node with degree 2 and compute the effective coupling $J_{\text {eff }}$ between its neighbors as a function of the temperature, by partially summing the partition function of the three-spin system over the states of the central spin. Assuming $J=1$, taking the average over the bimodal RF with amplitude $\theta$, we obtain $J_{\text {eff }}=c \psi(\theta, D) / 2 \beta, \psi$ being defined by Eq. (16). Some representative curves of $J_{\text {eff }}$ versus $T$ are shown in Fig. 5. For a constant $\theta=0.3$, and $D>0.5$, in the upper panel of that figure, there is a null effective coupling at a low temperature. So the existence of low-degree nodes, associated with a large crystal field, prevents the appearance of a long-range order. As the effective coupling increases with the temperature, a reentrant NM to F transition is allowed to take place. As the average connectivity increases, the number of low-degree nodes decreases, resulting in a less pronounced reentrant behavior, as we can conclude by comparing the $T / J$ versus $\theta / J$ phase diagrams for $c=5$ and $c=10$, in Figs. 4(a) and 4(b), respectively. This means that the reentrant behavior is clearly related to the finite connectivity and cannot be captured by a fully connected, mean field theory. The known mean field result that shows no reentrance is retrieved when $c \rightarrow \infty$ [23]. On the other hand, a large RF favors the active states. Consequently, for large $\theta$ the effective coupling is monotonically decreasing as a function of $T$, as shown in the lower panel of Fig. 5. This means that the $\mathrm{P}$ to $\mathrm{F}$ transition, which occurs in the high- $\theta$ side of the F phase, is not reentrant, even for low $c$.

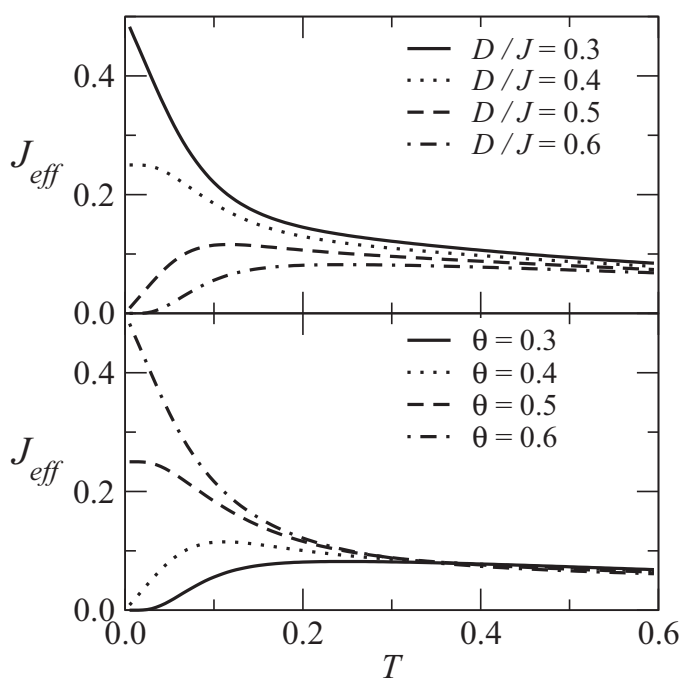

FIG. 5. Effective coupling between the neighbors of a node of degree 2 as a function of the temperature. Upper panel for several values of $D$ and constant $\theta=0.3$; lower panel: for several values of $\theta$ and $D=0.6$.

\section{SUMMARY AND CONCLUDING REMARKS}

The equilibrium statistical mechanics of the BC model in a random network with finite connectivity and a random local field that follows a bimodal distribution was investigated. The replica approach was applied to account for quenched disorder. The present approach, where it is possible to control network connectivity, allows us to vary, analytically, between a mean field theory to a low-connectivity theory that, although still in the infinite range, is closer to the real world, unveiling all the thermodynamic richness of the BC model.

In our work it was shown that the NM-F transition, at low connectivity, displays an inverse melting transition given by a reentrant behavior with a tricritical point in the reentrant branch. However, the amount of reentrance decreases when the connectivity increases and approaches the known mean field result, with no reentrance, as in the limit $c \rightarrow \infty$ [10]. Besides the small connectivity, the conditions for the reentrance are high crystal field and low-RF amplitude. So there is a reentrance in the NM-F transition, but there is not in F-P, as can be seen in Fig. 4. This results demonstrate that the topological mechanism for inverse melting in the RFBC model is active only for the NM phase and disabled for the $P$ phase. This distinct behavior can be explained in terms of a temperature-dependent effective coupling, which is more effective under low connectivity.

One of the most important findings of this investigation is that the mixed thermodynamic phase F/NM does not appear at low connectivity. This important result affects the subsequent phase diagrams when the crystal field $D$ is changing. As a consequence, the critical line between $\mathrm{F}$ and $\mathrm{F} / \mathrm{NM}$ is suppressed at low temperatures. When the connectivity increases, e.g., $c=25$ the mixed phase F/NM does appear. Nevertheless, the critical end point reported in the standard mean field approach still does not appear. The reason is that the F phase is suppressed, at low $\theta / J$, before the critical end point takes place. 
Despite the presence of quenched disorder, this study does not deal with frustration, since the couplings are always F. This means that the RS solution is stable, and no significant changes should be expected by changing to a replica symmetrybreaking solution.

A natural extension of this study could be to introduce disorder into the coupling constant $J$, which means to investigate the properties of the Blume-Emery-Griffiths [23] model in a random network.

\section{ACKNOWLEDGMENTS}

This study was supported, in part, by Conselho Nacional de Desenvolvimento Científico e Tecnológico (CNPq), under Contract No. 308501/2014-4 Brazil. The authors acknowledge J. J. Arenzon for useful discussions.

\section{APPENDIX}

In order to extract the variables under summation from the inner exponential in Eq. (6), we introduce the identity

$$
1=\sum_{\boldsymbol{\sigma}} \delta_{\boldsymbol{\sigma}} \boldsymbol{\sigma}_{i} \equiv \sum_{\boldsymbol{\sigma}} \prod_{\alpha=1}^{n} \delta_{\sigma^{\alpha} \sigma_{i}^{\alpha}} .
$$

Here $\sigma$ and $\sigma_{i}$ are vectors with $n$ components representing replica states and $\delta \sigma_{i}=1$ if $\sigma=\sigma_{i}$ and zero otherwise. The averaged replicated partition function becomes

$$
\begin{aligned}
& \left\langle Z^{n}\right\rangle=\sum_{\boldsymbol{\sigma}^{1} \ldots \boldsymbol{\sigma}^{n}}\left\langle\operatorname { e x p } \left[\beta \sum_{i, \alpha} \theta_{i} \sigma_{i}-\beta D \sum_{i, \alpha}\left(\sigma_{i}^{\alpha}\right)^{2}\right.\right. \\
& \left.\left.+\frac{c}{2 N} \sum_{i \neq j} \sum_{\boldsymbol{\sigma} \boldsymbol{\tau}} \delta_{\boldsymbol{\sigma}} \boldsymbol{\sigma}_{i} \delta_{\boldsymbol{\tau} \boldsymbol{\sigma}_{j}}\left(\mathrm{e}^{\frac{\beta J}{c} \sum_{\alpha} \sigma^{\alpha} \tau^{\alpha}}-1\right)\right]\right\rangle_{\left\{\theta_{i}\right\}} .
\end{aligned}
$$

Then we introduce the order function $P(\sigma)=(1 / N) \sum_{i} \delta \sigma \sigma_{i}$ through the identity

$$
\begin{aligned}
1= & \int \prod_{\sigma} d P(\sigma) d \hat{P}(\sigma) \\
& \times \exp \left\{\sum_{\sigma} \hat{P}(\sigma)\left[P(\sigma)-\frac{1}{N} \sum_{i} \delta_{\sigma} \sigma_{i}\right]\right\},
\end{aligned}
$$

where $\hat{P}(\sigma)$ is an auxiliary order function. Summing over $\sigma^{1} \cdots \sigma^{n}$ and changing $\hat{P}(\sigma)$ to $N \hat{P}(\sigma)$, Eq. (A2) becomes

$$
\begin{aligned}
\left\langle Z^{n}\right\rangle= & \int \prod_{\boldsymbol{\sigma}} d P(\boldsymbol{\sigma}) d \hat{P}(\boldsymbol{\sigma}) \exp N\left[\sum_{\boldsymbol{\sigma}} \hat{P}(\boldsymbol{\sigma}) P(\boldsymbol{\sigma})\right. \\
& +\log \sum_{\boldsymbol{\sigma}}\left\langle\mathrm{e}^{\beta \theta \sum_{\alpha} \sigma_{\alpha}-\beta D \sum_{\alpha} \sigma_{\alpha}^{2}-\hat{P}(\boldsymbol{\sigma})}\right\rangle_{\theta} \\
& \left.+\frac{c}{2} \sum_{\boldsymbol{\sigma} \boldsymbol{\tau}} P(\boldsymbol{\sigma}) P(\boldsymbol{\tau})\left(\mathrm{e}^{\frac{\beta J}{c} \sum_{\alpha} \sigma_{\alpha} \tau_{\alpha}}-1\right)\right]
\end{aligned}
$$

In the large- $N$ limit, the integral in this equation can be evaluated by the saddle-point method. The free energy is given by the extreme

$$
\begin{aligned}
f(\beta)= & -\lim _{n \rightarrow 0} \frac{1}{\beta n} \operatorname{Extr}\left[\sum_{\boldsymbol{\sigma}} \hat{P}(\boldsymbol{\sigma}) P(\boldsymbol{\sigma})\right. \\
& +\log \sum_{\boldsymbol{\sigma}}\left\langle\mathrm{e}^{\beta \theta \sum_{\alpha} \sigma_{\alpha}-\beta D \sum_{\alpha} \sigma_{\alpha}^{2}-\hat{P}(\boldsymbol{\sigma})}\right\rangle_{\theta} \\
& \left.+\frac{c}{2} \sum_{\boldsymbol{\sigma} \boldsymbol{\tau}} P(\boldsymbol{\sigma}) P(\boldsymbol{\tau})\left(\mathrm{e}^{\frac{\beta J}{c} \sum_{\alpha} \sigma_{\alpha} \tau_{\alpha}}-1\right)\right]
\end{aligned}
$$

over the set of densities $\{P(\sigma), \hat{P}(\sigma)\}$. The saddle-point equations are

$$
P(\boldsymbol{\sigma})=\frac{\left\langle\mathrm{e}^{\beta \theta \sum_{\alpha} \sigma_{\alpha}-\beta D \sum_{\alpha} \sigma_{\alpha}^{2}-\hat{P}(\boldsymbol{\sigma})}\right\rangle_{\theta}}{\sum_{\boldsymbol{\sigma}}\left\langle\mathrm{e}^{\beta \theta \sum_{\alpha} \sigma_{\alpha}-\beta D \sum_{\alpha} \sigma_{\alpha}^{2}-\hat{P}(\boldsymbol{\sigma})}\right\rangle_{\theta}}
$$

and

$$
\hat{P}(\boldsymbol{\sigma})=-c \sum_{\boldsymbol{\tau}} P(\boldsymbol{\tau})\left(\mathrm{e}^{\frac{1}{c} \beta J \sum_{\alpha} \sigma_{\alpha} \tau_{\alpha}}-1\right) .
$$

Eliminating $\hat{P}(\sigma)$ in Eq. (A6) by means of Eq. (A7) we obtain the self-consistent relationship (8).
[1] Y. Imry and S.-K. Ma, Phys. Rev. Lett. 35, 1399 (1975).

[2] M. Aizenman and J. Wehr, Phys. Rev. Lett. 62, 2503 (1989).

[3] K. Hui and A. N. Berker, Phys. Rev. Lett. 62, 2507 (1989).

[4] R. Monasson, J. Phys. A: Math. Gen. 31, 513 (1998).

[5] M. Blume, Phys. Rev. 141, 517 (1966).

[6] H. W. Capel, Physica (Utrect) 32, 966 (1966).

[7] N. Schupper and N. M. Shnerb, Phys. Rev. E 72, 046107 (2005).

[8] J. A. Plascak, J. G. Moreira, and F. C. S Barreto, Phys. Lett. A 173, 360 (1993).

[9] P. D. Beale, Phys. Rev. B 33, 1717 (1986).
[10] M. Kaufman and M. Kanner, Phys. Rev. B 42, 2378 (1990).

[11] S. K. Ghatak and D. Sherrington, J. Phys. C 10, 3149 (1977).

[12] A. Malakis, A. Nihat Berker, I. A. Hadjiagapiou, N. G. Fytas, and T. Papakonstantinou, Phys. Rev. E 81, 041113 (2010).

[13] P. E. Theodorakis and Nikolaos G. Fytas, Phys. Rev. E 86, 011140 (2012)

[14] F. P. Fernandes, Douglas F. de Albuquerque, F. W. S Lima, and J. A. Plascak, Phys. Rev. E 92, 022144 (2015).

[15] D. De Martino, S. Bradde, L. Dall'Asta, and M. Marsili, Eurphys. Lett. 98, 40004 (2012).

[16] L. Viana and A. J. Bray, J. Phys. C: Solid State Phys. 18, 3037 (1985). 
[17] I. Kanter and H. Sompolinsky, Phys. Rev. Lett. 58, 164 (1987).

[18] M. Mezard and G. Parisi, Europhys. Lett. 3, 1067 (1987).

[19] K. Y. Wong and D. Sherrington, J. Phys. A: Math. Gen. 21, L459 (1988).

[20] M. Mézard and G. Parisi, Eur. Phys. J. B 20, 217 (2001).
[21] T. Nikoletopoulos, A. C. C. Coolen, I. Pérez Castillo, N. S. Skantzos, J. P. L. Hatchett, and B. Wemmenhove, J. Phys. A: Math. Gen. 37, 6455 (2004).

[22] R. Erichsen Jr. and W. K. Theumann, Phys. Rev. E 83, 061126 (2011).

[23] M. Blume, V. J. Emery, and R. B. Griffiths, Phys. Rev. A 4, 1071 (1971). 